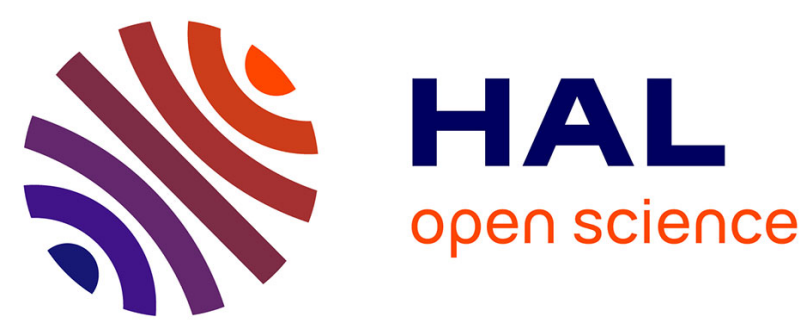

\title{
Visual Analytics of Political Networks From Face-Tracking of News Video
}

Benjamin Renoust, Dinh-Duy Le, Shin'Ichi Satoh

\section{To cite this version:}

Benjamin Renoust, Dinh-Duy Le, Shin'Ichi Satoh. Visual Analytics of Political Networks From Face-Tracking of News Video. IEEE Transactions on Multimedia, 2016, 18 (11), pp.2184 - 2195. 10.1109/TMM.2016.2614224 . hal-01454822

\section{HAL Id: hal-01454822 \\ https://hal.science/hal-01454822}

Submitted on 3 Feb 2017

HAL is a multi-disciplinary open access archive for the deposit and dissemination of scientific research documents, whether they are published or not. The documents may come from teaching and research institutions in France or abroad, or from public or private research centers.
L'archive ouverte pluridisciplinaire HAL, est destinée au dépôt et à la diffusion de documents scientifiques de niveau recherche, publiés ou non, émanant des établissements d'enseignement et de recherche français ou étrangers, des laboratoires publics ou privés. 


\title{
Visual Analytics of Political Networks from Face-tracking of News Video
}

\author{
Benjamin Renoust, Member, IEEE, Dinh-Duy Le, Member, IEEE, and Shin'Ichi Satoh, Member, IEEE
}

\begin{abstract}
The rich nature of news makes it a classic subject of visual analytics research. Such analysis is often based on rich textual data. However, we want to test how much we can understand the news from video information through face detection and tracking. Towards this goal, we propose a visual analytics system and discuss its design and implementation to support media experts in understanding political interactions in an archive of twelve years of the Japanese public broadcaster NHK's News 7 program. After identifying the tasks and abstraction required for our analysis, we construct links from face detection and tracking to derive multiple political networks. Our proposed design embeds this rich data into a visual analytics framework that presents four levels of abstraction: time period, network, timeline, and face-tracks within video. We present how the exploration of the archive with our system results in good understanding of the Japanese politico-media scene during these twelve years while finding evidence of "presidentialization" of the media.
\end{abstract}

Index Terms-Data visualization, Multimedia database, Graph, Face recognition.

\section{INTRODUCTION}

Computer science provides now quantitative capability for news analysis, empowering sociology and media studies. News monitoring has been addressed since the early 2000's in the discipline of topic detection and tracking [1]. It aims at segmenting, identifying, and following information, mainly from raw textual information. However, news videos are a very popular way of consuming news, and the images they convey have a powerful impact on viewers [2].

Working with a domain expert, we are motived by the action of understanding of how one can understand the news from its images, and how the media can be influenced by politics. If we use the image information, what can we understand from the politics portrayed by the media? Inspired by recent studies in social science [3], the following general questions drove our study: How is the opposition represented during the different government cabinets? Can we find traces of the "presidentialization" in the media? We propose to find elements of answers by exploring twelve years of the Japanese broadcaster NHK's News 7 program with a visual analytics system designed for our domain expert.

In a previous study [5], we discussed our investigation on how we could automatically form social networks from face

B. Renoust, D. Le and S. Satoh are with the National Institute of Informatics (NII), Tokyo Japan. B. Renoust is also with the Japanese-French Laboratory for Informatics (JFLI) CNRS UMI 3527, Tokyo, Japan. E-mails: \{renoust, ledduy, satoh\}@nii.ac.jp; tel:+81342122410; fax:+81342122120

1 "Presidentialization" is defined as an "increasing [media] attention to the rising importance of prime ministers" - as opposed to their own party [4]. detection and tracking in video, and which networks can be relevant to lead to a political analysis of the Japanese news. This was only a preliminary step that explored all possible networks we could generate, which is necessary to validate which data abstraction would be relevant to our problem. In this study, we integrated these networks into a visual analytics system and put their exploration at the center of the analysis of our 12-year archive.

Our contribution is a visual analytics system design and its implementation. The design supports the political analysis of a large-scale news video archive based on face-tracking, i.e., without text analysis. The data consists of 12 years of NHK's News 7 program with meta data. In coordination with our domain expert, we identified tasks and previously validated a data abstraction that is based on our video extracted data, combined with the expert's political data - the meta data which drives the analysis. Our implementation consists of four coordinated levels of abstraction: an entry point by the Prime Minister's cabinets, political social networks, timelines, and face-tracks within videos. We present results on how the use of our design by our expert led to the understanding of main events in the politico-media scene of NHK. The design of the system can help other researchers to tackle similar questions on different datasets.

After discussing related work in the next section, we will present our tasks and data in Section III. In Section IV, we introduce our data abstraction and summarize our methodology. In Section V, we present our visual analytics design and implementation regarding the identified tasks. In Section VI, we give insights derived from our domain expert's visual exploration of the 12 years of NHK's News 7 program. We discuss our observations, possible improvements of our design, and future work in Section VII before concluding.

\section{RELATED WORK}

News analysis has been a main target for visual analytics applications, for which the literature is prolific. The majority of related work explores text-based news data ([6], [7], [8], [9], [10], [11], [12], [13], [14], [15]), the focus of which was often on event and topic analysis, to reveal stories to users. Although we wanted to differentiate our study from these by solely focusing on the cues we extract from the visual information of the videos, we also incorporated political affiliations in our design. It is important to note that the related studies somtimes incorporated external data such as demoscopic information [15] or sentiment analysis [7] sometimes in combination with geographical information [6]. 
While some studies were aimed at supporting the understanding of news stories by delivering a summary of their content ([16], [13], [8], [6]) others were aimed at delivering the actual content depending on users' analysis ([17], [9], [10], [18]). Incidentally, the latter case appears mostly with systems involving video information, whether users are experts or not, for whom watching the content is often a necessary task. We propose a hybrid system of both exploration and content understanding. Our system drives the video exploration from a social analysis perspective. After understanding the situation and finding interesting insights, experts will be able to refer to the videos to further understand the relationship between politicians.

It should also be noted that the studies by Luo et al. [16], Itoh et al. [18] and Ide et al. [15] involved the same dataset we used with different purposes. Luo et al. [16] presented users video excerpts from a news story, and the users could hop from one video to another based on their interests. This exploration focuses on users interest, so the visualization accurately presents a neighborhood of keyframes, but does not provide any overview of the data. Itoh et al. focused on transmedia exploration and presented timelines of clustered keyframes in a 3D space. Interaction is aimed at finding the source documents of specific events. Ide et al. [15] automatically constructed summary videos explaining major news events.

Many visual analytics systems are focused on the coordination of timelines ([18], [10], [6], [8], [7], [9]). They often include the representation of the topics themselves, e.g. through the distribution of keywords or keyframes. NewsRover [9] additionally displays actors when users watches a video that allows on to jump to actors mentions. Although we do not exploit topical information, time representation is a necessary aspect of our system design.

Co-occurrences in the news has led to the production of many social network analyses and visualizations, and the relevance of network modeling for political studies no longer needs to be proven [19]. Co-occurrences are mostly extracted from textual information. In particular, character networks have been broadly analyzed from literature [20], and TV dramas [21], and there is even a website dedicated to the social analysis of Game of Thrones ${ }^{2}$. The topological analysis of the networks is a key component in this type of analysis. This aspect has been investigated with varying focus. Matrix-based visualizations are often used for social networks visualization ([22], [23]) but we follow Ghoniem et al.'s recommendations [24] to use node-link diagrams because we want to observe the topological structure as well as exploring neighborhoods in reasonably sized graphs.

When political networks are too dense, an emphasis on edge bundling has been proposed to highlight interactions between politicians [12]. Networks of names [11] enables a neighborhood exploration of named entities. Tagesnetzwerk [14] presents a hierarchical exploration of an aggregated graph of stories formed from named entity clusters. Detangler [13] uses the coordination of two networks to reduce the visual

${ }^{2}$ https://gameofnodes.wordpress.com/ complexity induced by multiple types of entities and relationships in the semantics extracted from news. We introduced network analysis from video analysis [5] - although we found an interesting effort in generating networks of multimedia content for digital forensics [25] - and our previous study [5] was aimed at validating the relevance of this method for political network analysis during the early steps of our design.

Our system is focused on faces detected in news video, for which new deep learning approaches are very promising. Face recognition has now become extremely accurate ([26], [27]), and robust against many transformations and postures [28] and occlusion and lighting conditions [29]; however it is sensitive to erratic motions. All the previously mentioned approaches require many training sets with well-tuned parameters on a large infrastructure before being able to be deployed in the wild; however, they will been soon available to everyone so we should consider their applications to real-world datasets. We use an integrated approach optimized from large-scale applications [30], although it is not as accurate as the aforementioned approaches.

\section{TASKS AND DATA}

Following Seldmair's et al. guidelines [31], we now describe the tasks and dataset that motivated our design.

\section{A. Identifying tasks}

The system we propose is for an expert in media studies who is interested in exploring how purely visual information can help understand the news in Japan. He gave us the question: What could be revealed from politicians appearing in the news over the years? In fact, our expert was motivated by some specific high level questions such as How is the opposition represented during the different government cabinets? Can we find traces of "presidentialization" of the politics in the media? The case of the NHK is especially interesting because it is a national channel, i.e. influenced by the government, from which we want to see the impact on the politicians' appearances. Together, we identified the following necessary tasks for our visual analytics system:

T1: display the politicians appearing in the news,

T2: display the relationships between politicians in the media, T3: display their (co)appearance over time,

T4: identify "outstanding" individuals,

T5: differentiate between parties and leaders in power,

T6: give an overview of the politician/party interactions during the different cabinets,

T7: identify the role of a party during a specific cabinet, T8: allow comparison across cabinets (globally, from individuals, and relationships),

T9: allow investigation and comparison of individual relationships and appearances during a cabinet,

T10: identify corresponding video segments for viewing, T11: access the video segments from individual face-tracks, T12: locate and display face-tracks in video segments for validation. 


\section{B. Dataset}

The video dataset we analyzed is a daily collected archive of NHK's News 7 [32]. The archive has been added to since March 17, 2001, and the data we used stop on February 27, 2013: 2,102 hours of archived videos. A news program is typically composed of different stories, which we will call news segments (Fig. 1-(1)). The meta-data consisting of newssegment boundaries were kindly provided by Ide et al. [33].

The time period observed in our data is particularly interesting to our domain expert in regards to the government's instability. The two main Japanese opposing parties (the Liberal Democratic Party, hereafter LPD, and the Democratic Party of Japan, hereafter DPJ) have been ruling the country through 11 cabinets and 8 Prime Ministers. The capture period starts with over eight years of LDP leadership, followed by about three years of DPJ control, before switching back to the LDP, with almost a different cabinet each year. A new cabinet is formed every time a Prime Minister changes his government: after three cabinets spanning over five years of the very popular Jun'Ichiro Koizumi, six different Prime Ministers have each led a 1-year cabinet before Shinzo Abe (the current Prime Minister) came back into power.

This allows us to create 11 time-frames, one for each of the cabinets covering our capture period, and to compare them one another (T5 to T9, Figs. 4 to 12). Two time periods cover a very small portion of the capture, the pre-Koizumi cabinet, led by Yoshiro Mori and the second cabinet of Abe (at the end of our capture), only overlapping by a few weeks. In addition, our domain expert provided us a list of 40 Japanese politicians with their political affiliations (T5 to T7). Our preliminary analysis (see below) resulted in detection of other actors in the news (celebrities, athletes, international figures, etc.), and we included an additional 35 foreign politicians.

\section{DATA ABSTRACTION}

We need to monitor the activity of the politicians in the news (all tasks) and derive their relationships for social network analysis and exploration (T2, T6, T8, T9). Our first intermediary step was to validate which abstraction would be sufficient for political analysis. This was the main focus of our previous study [5]. We now summarize the methodology we used to derive and choose the right data abstraction.

\section{A. Face tracking}

We followed the method by Ngo et al. [30] to identify the different actors in the news segments. It allows face-tracking and identification following four steps: face detection, facetracking, sampling, and face-track matching, after which we went through face-track annotation.

Face detection (Fig. 1-(2)): We first apply the Viola-Jones face detector [34] to every frame of our videos. The detection results in a list of face-boundaries in an image, some of which may overlap.

Face tracking (Fig. 1-(3)): We then apply the KLT point tracker [35] to each face-feature point. The point tracks are matched given their temporal order from a starting face to the

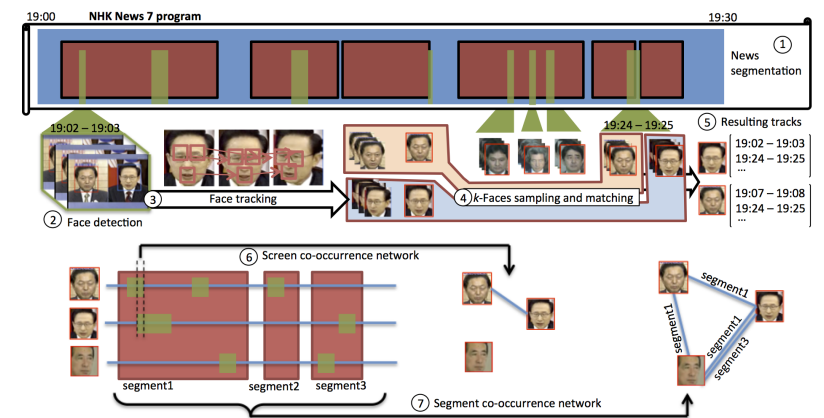

Fig. 1. Framework of our news video analysis. (1) News segments are identified. (2) Faces are detected in each frame. (3) Point tracks are inserted and matched across faces creating face-tracks. (4) Tracks are sampled and clustered to obtain the final face-tracks for each actor (5). (6) Across all segments of all news, co-occurrence links are extracted to form networks. (7) News segments can be used to form multiplex networks.

following faces, resulting in multiple face instances regrouped in face-tracks.

Face sampling (Fig. 1-(4)): Each face-track is compressed into one representative face feature representation. The $K$ faces are sampled for each track [30] and averaged in their VGG feature space [36].

Face-track matching (Fig. 1-(4)): The representative faces can be matched given their Euclidian distance in the feature space. During one week, the retrieval results were manually annotated (name, country and occupation) to obtain 139 actors, making sure that our politicians were included.

The entire processing took about 3 weeks on our $4 \mathrm{CPU}$ Intel Xeon X7560 server. The resulting data consist of a video per day, for which we have news segments, and a list of different actor's face-tracks with face boundaries (Fig. 1-(5)). Finally, we obtained 12 million faces and 16,714 face-tracks of annotated data, with 1.28 actors per segment on average. Thankfully, although the final data were relatively sparse given the 12 years, this shows reasonable expectation for actors to overlap across news segments.

As we discussed in our previous study [5], our face-track analysis provides multiple ways to measure an actor's activity in the political landscape formed by the news. The first measure, maybe the most natural one, is "airtime", i.e., how long a person appears on screen. A second measure we call "coverage", is the number of news segments in which an actor appears. This tells how many stories concern a particular actor over an observed period. Airtime is the measure of choice of our domain expert because of its obvious interpretation; however, it is still unreliable (as we discuss in Section VII). We obtained a Pearson's correlation coefficient of 0.95 between both measures over all actors. Because they are highly correlated, we agreed that coverage, which relates to our definition of links (see the following section), will find its way in our visual encoding (see Section V).

\section{B. Deriving links}

The first links that naturally interest our domain expert were made from screen co-occurrence, i.e., when two actors appear simultaneously on screen (Fig. 1-(6). This makes it 
possible to measure the co-appearance airtime. Unfortunately, as discussed in our previous publication [5], the data obtained were too sparse, and this network is by far not representative enough to conduct our visual analysis. However, we will keep this as a property of our links (Fig 2-(c)).

Nonetheless, we concluded [5] that news segmentation can be used as an alternative for uncovering links across actors. When two different actors appear in the same news segment, this means that they took part or shared interest in the same media event. Based on this intuition, we can derive a link between two politicians when both appeared during the same segment (Fig. 1-(7)). We finally obtained a network through 920 news segments, connecting our 75 politicians with 397 links across 1875 interactions over time - these are the links only, the politicians themselves appeared in 4418 unique news segments.

We end up with multiple links, each occurring over time, forming a multiplex network that we can look at from the perspective of each different cabinet. Each node and each link represent a time series corresponding to excerpts of news video with face-tracking. The topology of the network, the position of a node in the network, its coverage, and the coverage of the links will then vary from one time period to another. Our domain expert chose this abstraction to serve as a base to compare the activity of politicians across cabinets.

\section{A Multi-level Visual Exploration}

Our domain application is the political analysis of the different cabinets of Prime Ministers. Following Schneiderman's famous mantra [37] "overview first, zoom and filter, then details on-demand", we conduct a visual exploration of the data from a multi-level perspective. The first level of analysis is a preview of these cabinets in an aggregated manner. The second level of exploration enables us to dive into the social networks taking place into the cabinets. The third level locates the graph elements in time. The last level enables us to conduct a detailed exploration of the videos and face-tracks. We discuss our design choices given the tasks we identified in Section III-A and Brehmer and Munzner's why, how, what model [38] with the taxonomy they used.

\section{A. Cabinet view}

We first offer a network oriented visualization that allows comparison between the different cabinets (Fig. 2-(1)). It is the first entry point to access the different social networks.

Why: Present, summarize, compare, discover This view addresses T5 and mainly T8. Its first goal is to locate the different cabinets in time with some basic properties, the dominant party, and political actors. Then it aims to relatively compare the different cabinets in term of the presence or absence of the main political actors. It basically helps in providing an overview of how similar the politico media scenes are in terms of politicians' appearances.

How: Aggregate, encode, select, filter, derive In this view, each node represents a cabinet, the links between nodes display the proximity between cabinets in terms of their main political actors. The main actors form a subset of notable politicians given the following criterion: they can correspond to either the Prime Minister, or appear in the top 5 (arbitrarily chosen) in coverage and between-ness centrality in a cabinet network. This gives us a total of 28 notable politicians over our 11 time periods. This prevents noise generated by marginal politicians when we compute the Jaccard proximity between cabinets.

With the knowledge of the party in power, we embed the results in a circular node-link layout. Our choice of a nodelink layout - when a matrix representation is more common for similarity graphs - may go against the recommendations from Ghoniem et al. [24], but the visual complexity is manageable due to the limited number of time periods. We take advantage of the order constrained by time to lay out the nodes in clockwise order, placing the first period at the topmost position (as reflected by their label). Size and color encodings (following a ColorBrewer yellow-orange linear scale [39]) correspond to the number of main actors in the graph for nodes and to the proximity between nodes for the edges. The border color of a node corresponds to the party leading the cabinet (red for the $D P J$, and blue for the $L D P$, which is important for T5). This layout is made purely for aesthetically pleasing reasons implied by the relation to time. When we will need to include more cabinets we will switch to a matrix-based representation.

A selection in this graph triggers the next level of exploration. It opens a new view for each selected node, each corresponding to a time period of the social network. Additionally, the newly derived views come with highlighted nodes corresponding to the shared main actors (helpful for T4). In a similar manner as with Detangler [13], this selection can be tuned to correspond to a union or intersection of actors across the different edges.

What: to generate the view; In: multiple networks from each time period; Out: similarity network.

What: for the dive-in interaction, it is actually the reversed operation from the what that creates this view; In: a selection of nodes/edges in the similarity network (Fig. 2-(a); Out: multiple network views with highlights corresponding to the selection (Fig. 2-(2), 2-(3)).

\section{B. Network views}

After making a selection in the cabinets view, the user is given new additional views, one for each selected cabinet (Fig. 2-(2), 2-(3). The core analysis of the data takes place in these views, as they address many tasks.

Why: Present, search, identify, compare

The social network analysis will be conducted in this view. The first goal with this view is to display the politicians the news (T1) and identify specific actors (T4) and relationships (T2). Given a network, we need to understand its global topology (T6) then compare it in different time periods (T8). The view presents a network with multiple encodings of the characteristics of our data that displays the coverage of politicians/relationships (T9) and allows the quick identification of party affiliation (T7). Linked-highlighting allows comparison, from links between parties within one network (T7), between individual politicians and relationships across parties (T8). 


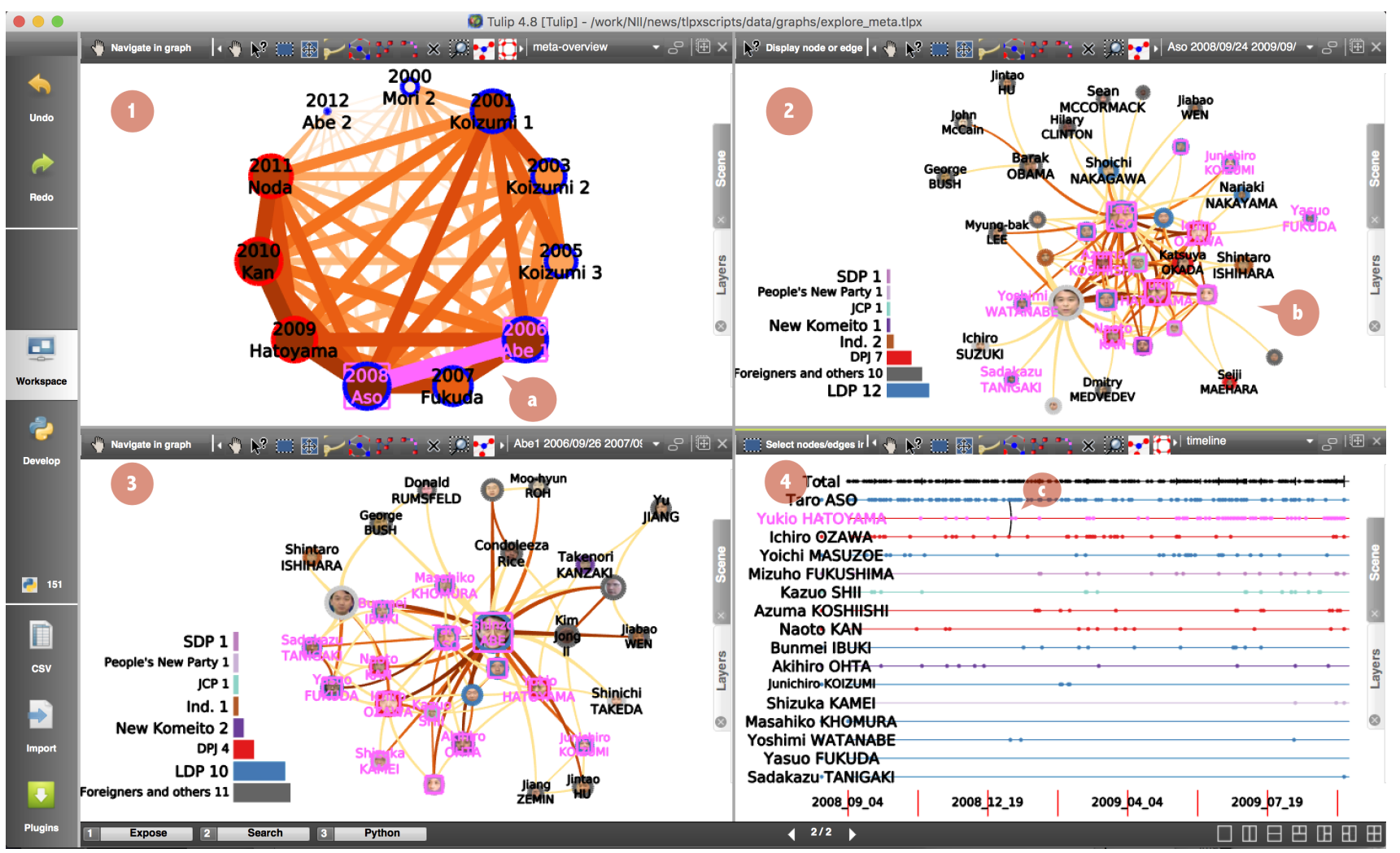

Fig. 2. Details readable in zoom. Framework for our network exploration within Tulip. User starts from selection in cabinet view (1). Link is selected - (a) (in pink) - across two cabinets (Abe 1 and Aso, both from LDP) which shares most political actors. For each cabinet, two network views are opened (2), (3). Common actors are highlighted (in pink). During Abe's first cabinet (3), topology of network is centered and highlighted actors are very much centered around Prime Minister Abe, however, during Aso's cabinet (2), links between selected actors form tight clique. Timeline view (4) was derived from selection in Aso's cabinet (b), showing activity of different actors during cabinet. Of course, Prime Minister Aso is shown in most of media activity during timeline. Hatoyama represents main opposition leader during cabinet and displays intensifying activity at end of time period (second line in (4)): he will actually be next Prime Minister. His position in Abe l's network is rather peripheral, contrary to Aso's network in which he is at heart of main clique. Incidentally, screen co-occurrence between Ozawa and Aso (c) can be immediately identified and accessed.

How: Encode, navigate, select, derive

We chose a navigable node-link layout, which is, this time, fully justified following the recommendations from Ghoniem et al. [24]. Nodes are actors (T1) and links are created if there is a link in the original multiplex network connecting two actors during the current time-period (T2). Topological observations allow for identification and comparison tasks (T4, T7, T8), and the visual complexity of each network is manageable. The visual encoding of the nodes consists of three pieces of information: the size reflects the weight that can be set to be the node's between-ness centrality, or the actor's coverage (T4); the texture is the image of the actor attributed to the node (T1), and the border color of the node reflects the political affiliation of the actor (T5, T7). The graph is laid out using the GEM force-directed algorithm which works well in minimizing edge crossing [40]. The links encode the co-appearance of politicians during the cabinet, as reflected by their color (linearly mapped using a ColorBrewer yelloworange scale).

Users can of course switch the encoding of the node size between centrality and coverage (T4). If links are selected in the previous cabinet view, nodes may already be highlighted (T4). Nodes and links are linked-highlighted across all different network views, so that the user can investigate and compare the respective position and characteristics of nodes and links in different time-periods (T8).

An additional caption is attached to explain the mapping between color and political affiliation. It is represented as a mini-horizontal bar chart to reflect the distribution of the different party members during the cabinet. Selecting a party in the caption highlights the nodes of actors that have affiliation during the time period, along with the links between these actors. If multiple parties are selected, the effect is the same but limited to the links connecting members between parties (T7). From a selection of nodes or links, the user triggers a dive-in interaction to open the next level of visualization and display the corresponding timelines (T3).

What: for the dive-in interaction, we obtain the data corresponding to news segments in the multiplex network; In: a selection of nodes and links in a network (Fig. 2-(b)); out: time-series view of the corresponding occurrences (Fig. 2-(4)).

\section{Timeline view}

The timeline view allows for a detailed time-series exploration of the selected nodes and links (Fig. 2-(4)).

Why: Present, search, compare, produce

In this visualization, we are looking for specific patterns (T4) or points in time. The goal is to explore a timeline to 


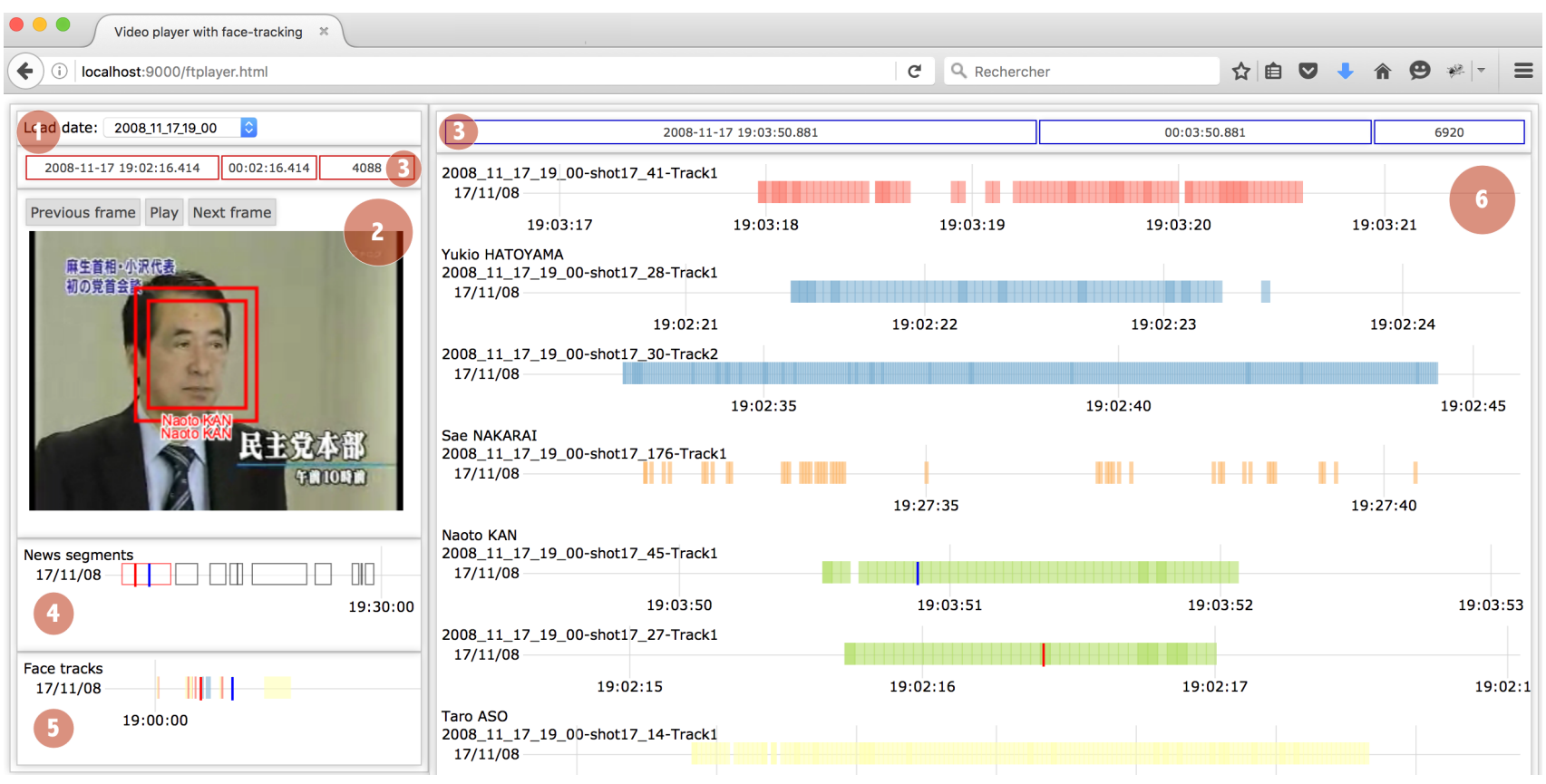

Fig. 3. Details readable in zoom. Web-embedded player. (1) Date is chosen in playlist. (2) Video player allows frame-by-frame control, with overlaid face boundaries. (3) Time indications for both cursors (red, player's current position, blue, mouse pointer's position). (4) Time of different news segments (selected segment highlighted). (5) Timeline of different detected face-tracks. (6) Face-tracks for each different actor. Notice how cursors are synchronized across "news segments", and "facetracks" timelines. Darker bins in face-track timeline signify multiple detections of same face, as current video position and face boundaries in player show.

investigate actors' (co)appearances (T3). We offer to locate actors' (co)occurrences during the cabinet, monitor and compare screen activity (T9), and eventually identify specific news days to view related videos (T10).

How: Encode, navigate, select, derive

In a very classic way, parallel horizontal timelines are presented (oriented by time from left to right), for which each line corresponds to a selected node/link (T3). The lines are labelled and ordered vertically by their coverage (T4-T9). The color of a timeline recalls the color of the party presented in the previous subsection (helpful for T7). If the timeline corresponds to a link, the average color in the $R G B$ space is then computed. A bin positioned in the timeline corresponds to a day during the time span of the selection. The height of the bin encodes the number of different news segments detected that day. Bent links upon the timeline ([41], [42]) are drawn between bins to highlight the screen co-occurrence between two actors (T4). This encoding can be questioned whenlinks greatly increase, but as we discussed in Section IV-B, these links rarely occur, so the visual complexity they induce is manageable.

An additional timeline entitled "Total" cumulates all detections as a reference for comparison (T4). By selecting a node in this "Total" timeline, we can highlight the corresponding nodes in other timelines. From a selection in other timelines, users can trigger a dive-in interaction that opens our face-track player for further viewing and analysis of the corresponding video content (T10).

What: for the dive-in interaction, we identify segments of videos and tracking frames to feed the results to our facetrack video player; In: selected bins in a timeline (Fig. 2-
(4); Out: video-player with highlights corresponding to the selection (Fig. 3).

\section{Face-track video player}

This view is the final level of exploration. It consists of an augmented video player enriched with face-tracking information for detailed video access (Fig. 3).

Why: Present, search, compare

The goal is to navigate and refer to the videos. It is aimed at providing access to the video content directly from the face-tracks (T11). The video is consumed as usual with enriched face-tracking information (T12). Face-tracks can then be compared as the video plays (T12).

\section{How: Encode, select, navigate}

This visualization presents a video with controls (Fig. 3-(2)) together with multiple timelines (T11, T12). The first timeline entitled "News segment" corresponds to the news segments in the video (Fig. 3-(4)). Each bin located on the timeline encodes the timeframe of the news segment: a bin's width corresponds to its duration. Red segments are highlighted.

The second timeline entitled "Face-tracks" summarizes all face-tracks detected in the video (Fig. 3-(5)), useful for localization (T12). Similarly to the news segment, a facetrack is a video segment in which a face is detected. Since our face-tracks are not necessarily contiguous frame-by-frame, this summary only takes into account the min-max frame boundaries of a face-track. Each face-track of each different detected actor then corresponds to one bin in the timeline. The bin color encoding depends on the different actors (using a Brewer's qualitative scale); transparency is set so colors blend when bins are super-imposed. 


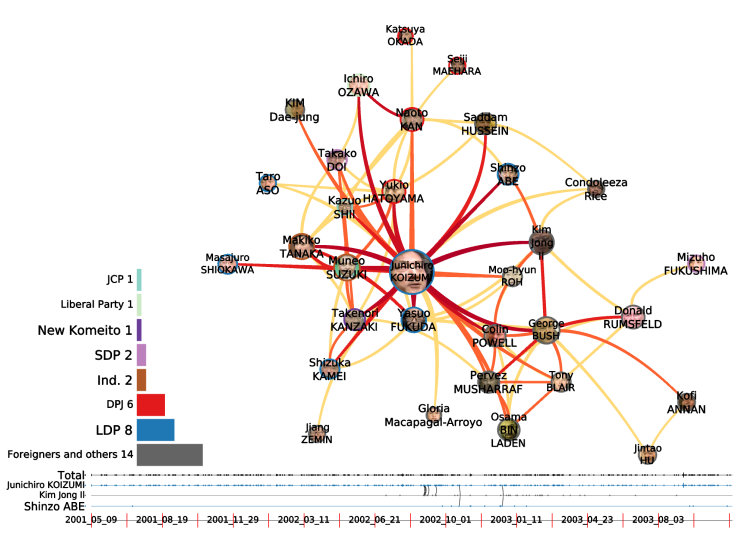

Fig. 4. Details readable in zoom. Koizumi's first cabinet (2001/4/26 2003/11/19), with comparison of early activity of Abe and Kim Jong Il.

We provide detailed face-tracks for each different actors (Fig. 3-(6) following the same encoding, and a timeline corresponds to one actor's tracking in the video (T12). A bin here corresponds to exactly one frame in the video. This enables one to look for holes in the face-tracking process potentially missing faces, and transparency makes it possible to locate when multiple faces are assigned to one actor.

All the timelines are navigable (pan and zoom), and two cursors make the linked-highlighting. The first red cursor corresponds to the current position of the video player (Fig. 3-(3) left), the precise time of day, time in the video, and frame number linked with the cursor are provided above, and its relative position is shown in every timeline (T12). The second blue cursor has exactly the same linking across the timelines and with the second time display (Fig. 3-(3), right). It corresponds to the position of the mouse while hovering over any timeline, placing on click the video to the hovered time. When the video is playing a frame that contains face-tracks, labelled face-boundaries are drawn on the video (T12).

\section{E. Implementation}

The first levels of exploration (Sections $\mathrm{V}-A, B$ and $C$ ) are built on top of the Tulip ${ }^{3}$ [43] framework. The views benefit from the full suite of Tulip's graph manipulation capabilities. This includes the ability to use different metrics for layout and coloring. We can also carry out graph theoretic grouping operations such as finding induced subgraphs and neighborhood selections. The framework also provides many useful complementary views not discussed in this paper, such as scatter plots and histograms, useful for comparing and searching tasks. The player (Section V.D) is built on top of $\mathrm{D}^{2}$ [44] for the timelines and synchronization, benefiting from all its interaction capability and flexibility, and uses HTML Canvas for the video and overlay.

\section{RESULTS}

The exploration of these networks led us to some understanding of the politico-media scene presented by NHK News 7. Our domain expert was very enthusiastic in using our

${ }^{3}$ available at http://tulip.labri.fr

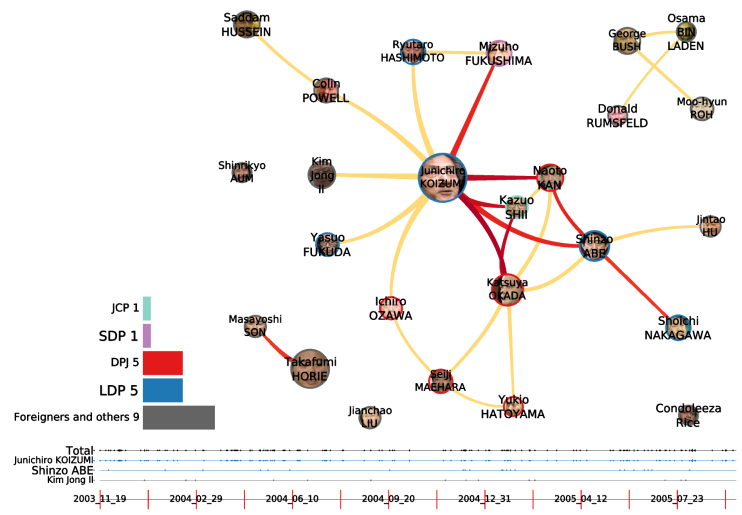

Fig. 5. Details readable in zoom. Koizumi's second cabinet (2003/11/19 2005/09/21), with comparison of activity of Abe and Kim Jong Il. Abe starts to grow in importance in network (node size and centrality).

system, and all the results below come from the observations and detailed exploration of the different cabinets done together with our domain expert. The following case studies were led by selecting, comparing, identifying, and drilling into timelines. Finally, our expert accessed some of the videos in the timeline to validate his understanding.

\section{A. Overview: different parties, different topologies}

From the overview of all cabinets (Fig. 2-(1)), we noticed a few interesting facts from looking at the links' weight. The first one is that the three cabinets of Koizumi are were becoming increasingly different from one another. Then, the politicomedia landscapes of the period when politicians played musical chairs (from Abel to Noda) did not have strikingly different politico-media scenes. The switch from the $L D P$ to the $D P J$ did not even result in dramatic changes: only Fukuda's cabinet appeared rather different during that period. We do not discuss the results from the observations of Mori and Abe2 cabinets due to their limited data, but they remain available to our domain expert.

Looking at the individual networks (Fig. 4 to 12), the first striking piece of evidence is that the different Prime Ministers stand out in our detection of NHK news. They appeared as most prominent nodes in all different measures of the data: their coverage was the maximum during their own cabinets and they also occuped the most central place in their network. There seems to be a trend in the concentration of media around the Prime Minister's activity during LDP cabinets, closer to "star-shaped" topologies (Fig. 4, 5, 7, 8 and 9), whereas interactions are much denser between DPJ members during their cabinets (Fig. 10, 11, and 12). The exception is the case of Koizumi's third cabinet (6), in which Abe was put in a central role, breaking the star-shape scheme.

We refer to a star-shape when the Prime Minister concentrates most of the links with other politicians showing much less interaction - the archetype being Koizumi's second cabinet (Fig. 5). This is in contrast with the case in which many politicians share many links, as displayed by Kan's cabinet (Fig. 11). This shows the difference in media strategies between both main parties. With the LDP, the Prime Minister 


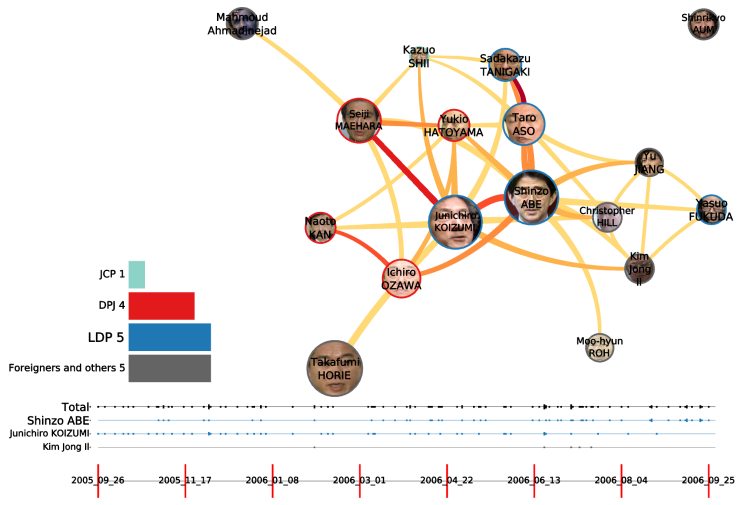

Fig. 6. Details readable in zoom. Koizumi's last cabinet (2005/09/21 2006/09/26), with comparison of Abe and Kim Jong Il's activities. Now Abe has even more prominent place than Koizumi (node size and centrality).

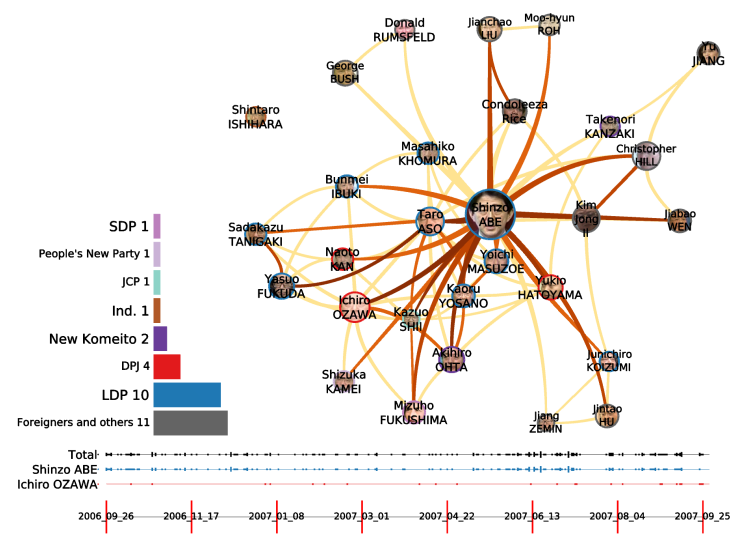

Fig. 7. Details readable in zoom. Abe's first cabinet (2006/09/26 2007/09/26), with comparison of his and Ozawa's activities. This is an archetype of "star-shaped" topology, with most weighted edges concentrated on most central node i.e. Abe.

is presented as a leader, i.e., a strong figure in charge of the country, whereas with the DPJ, the image is more of a board of leaders ruled by the Prime Minister. We have in this observation evidence of the "presidentialization" of Japanese politics in the media when the LDP is ruling. This result invites us to find measures of this phenomenon for future work.

\section{B. Game of cabinets}

We now report on the observations that were made during the network exploration with our domain expert. It was coordinated with timelines, allowing us to follow in detail the activities of politicians during each cabinet.

During Koizumil (Fig. 4), the structure of the links suggests that foreign affairs were important. The Prime Minister displays many connections with foreign politicians. During Koizumi's following two cabinets (Fig. 5 and 6), foreign politicians show less interaction with national politicians, and opposition members appear with higher coverage. The second cabinet of Koizumi (Fig. 5) shows a smaller network. Koizumi is the most central and most covered politician by far (largest node). A look at the timeline indicates that most coverage is concerned with his appearances. This confirms our expert's

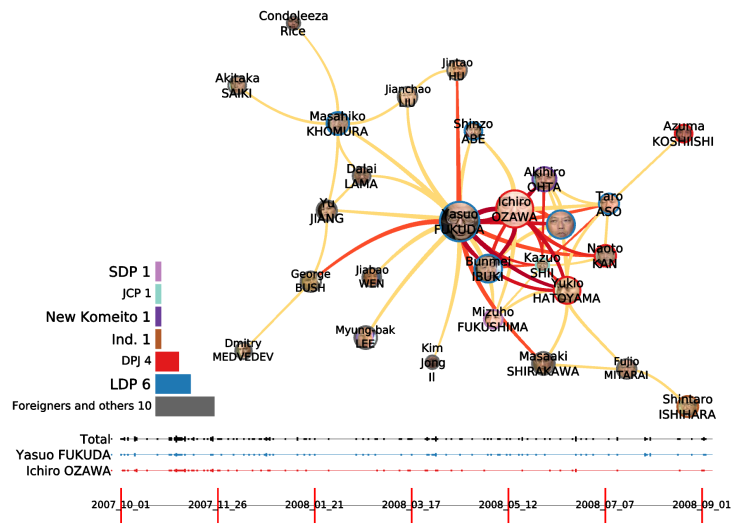

Fig. 8. Details readable in zoom. Fukuda's cabinet (2007/09/26 - 2008/09/24), with comparison to Ozawa's acitivity.

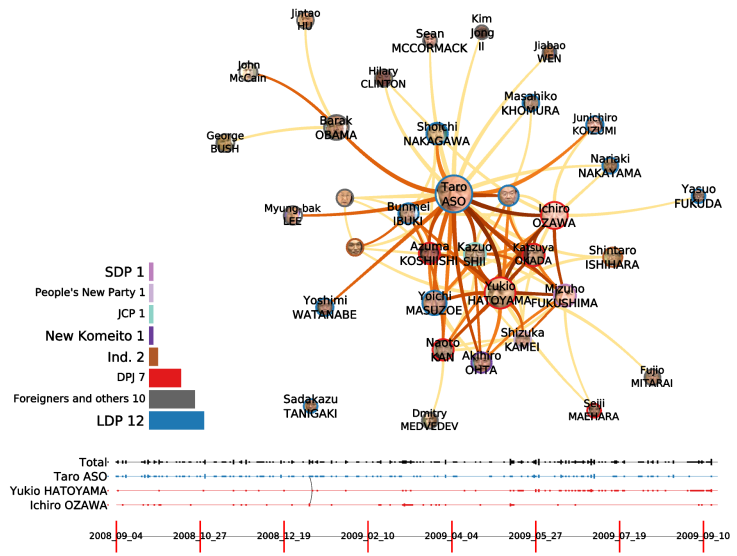

Fig. 9. Details readable in zoom. Aso's cabinet (2008/09/24 - 2009/09/16) with comparison of Aso, Ozawa, and Hatoyama's activities.

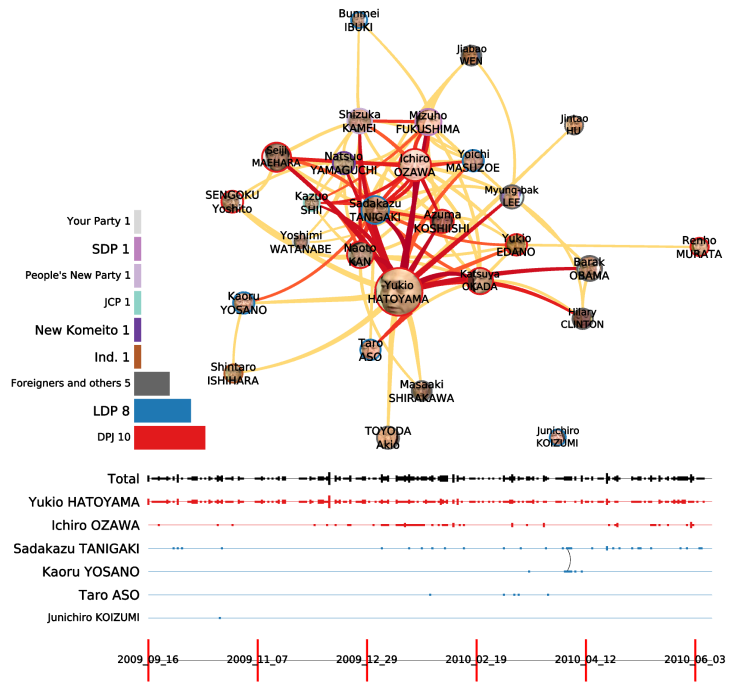

Fig. 10. Details readable in zoom. Hatoyama's cabinet (2009/09/16 2010/06/08), with comparison of Ozawa and all LDP members' activities.

knowledge giving evidence of the role of Koizumi in the "presidentialization" of Japan's politics [4].

The third cabinet of Koizumi (Fig. 6) clearly puts Abe in a 
extraordinary position in the network. He has strong links with opposition figures, even more than the Prime Minister in terms of connections with foreign leaders. Expected as the successor to Koizumi, the media covered him extensively, and Abe will turn out to be the next Prime Minister. The timeline exploration (from the selection of Koizumi and Abe) shows a growing appearance of Abe on screen, whereas Koizumi's appearance is decreasing. This reveals the strategy of the LDP to transition power to its exceptionally younger candidate, slowly putting him at the center of the news.

With linked highlighting, we can trace back Abe's appearance to late 2002. Watching related news then explains his growing popularity: he took strong actions to help free Japanese citizens abducted by North Korea - interestingly, Koizumi, Abe and Kim Jong Il's activities show some overlap during this period(Fig. 4).

Abe's first cabinet (Fig. 7) puts in perspective many LDP members and a varied opposition. Foreign politicians seem to be presented only in relation to the Prime Minister. One opposition leader, Ozawa, is particularly well connected with other politicians, either LDP or members of the opposition. Ozawa is sometimes (in)famously referred as the "Shadow Shogun"4. Put in short, he never ceased to try building alliances behind the scene, and often changed sides, to remain in an opposition role. From continuing to observe him playing a central position in the NHK politico-media scene (Fig. 8) and watching the news during Hatoyama's cabinet (Fig. 10, from a selection in the timeline during Hatoyama's cabinet), we can observe that he was put under investigation for corruption. Linked highlighting shows a dramatic change in his direct neighborhood in the following cabinets.

Prime Minister Aso's cabinet (Fig. 9) also displays many foreign leaders, and it signals the arrival of Obama as President of the USA. Although we can see strong co-occurrences between Ozawa and Aso, equally strong links connect Aso and Hatoyama of DPJ. The opposition leadership is actually changing during this cabinet, to the benefit of Hatoyama, which we can observe from the trends in the timeline of their links with the Prime Minister. Contrary to LDP members, the activity of DPJ members intensifies towards the second half of the cabinet. In fact, Hatoyama became the next Prime Minister and his activity stands out even five months before he was elected. It is during this transitional phase that we appear to have the most balanced representation between both main leaders in terms of coverage.

Kan's cabinet (Fig. 11) presents features similar to Hatoyama's cabinet. There is a much denser structure between DPJ members, with almost non-existent LDP members. Kan's cabinet witnessed the tragic Great East Japan Earthquake, and its consequences became the focus of the media, rather than political games. The timeline shows that most government members appeared after the tragedy on NHK. Discussions were controlled while it was preferred to put forward the actions of the government.

Finally, Noda's cabinet (Fig. 12) more resembles those of

\footnotetext{
${ }^{4}$ https://historyofjapan.wordpress.com/2014/12/20/episode-82-the-shadowshogun-redux
}

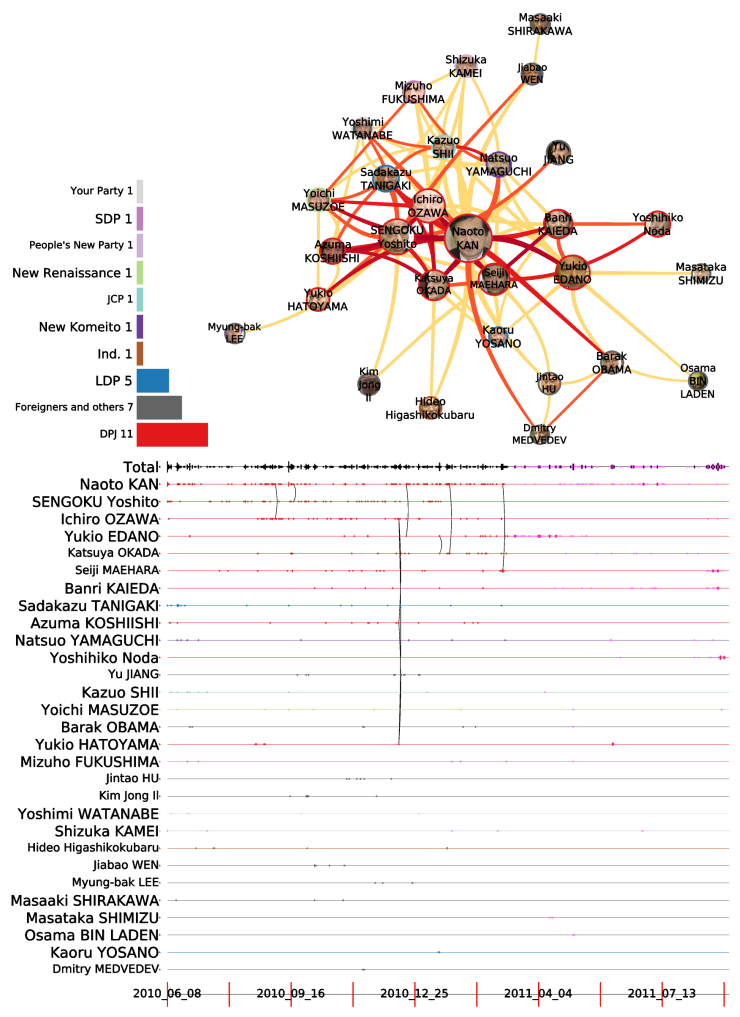

Fig. 11. Details readable in zoom. Kan's cabinet (2010/06/08 - 2011/09/02) with activity of all political players. Activity after Great East Japan Earthquake is highlighted. Network topology contrasts from "star-shape" as in Fig. 7, centrality and weighted links are more evenly spread across network.

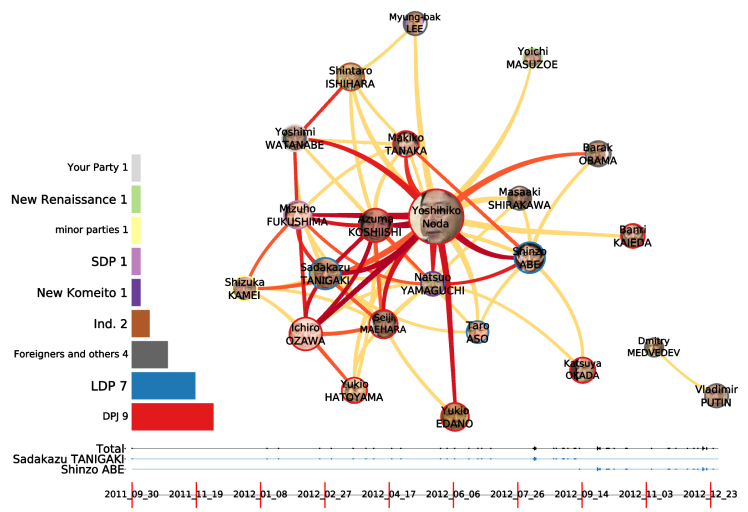

Fig. 12. Details readable in zoom. Noda's cabinet (2011/09/02 - 2012/12/26), and comparison of Tanigaki and Abe's activities.

Abe or Koizumi. The Prime Minister tends to hold a central position. The two main opposition figures, first Tanigaki and later Abe have quite separate positions in the network. The timeline clearly shows their transition. Again, it confirms our domain expert's knowledge at it was foreseen that DPJ would not win the next election. The interesting side effect is that many new parties begin to appear at the end of Noda's cabinet.

\section{Feedbacks on the face-tracking}

Because it would take a large amount of time to watch all the videos, the video player was used to isolate interesting videos and check whether the interpretation was correct. 


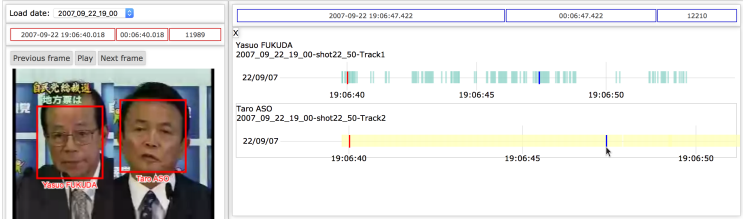

Fig. 13. Details readable in zoom. In this example, Aso and Fukuda globally cover same span of detection in case of split-screen illustrating an opposing ideas. Detailed inspection of their timelines shows many holes in detecting Fukuda.

Although our expert relied on the networks and timelines to formulate his interpretation, it was necessary to go down to the level of the video to verify that it was correct, by directly accessing a few videos. The face-tracks presented in our player greatly helped our expert to jump directly to the core segment he was interested in.

Using our video player, we also obtained some interesting overall feedback on the nature of face-tracks. It is often in the news that a politician is presented as being alone during some type of intervention (especially the Prime Minister). This may be an editorial choice, for which would need comparison with other channels. When several actors are presented on screen, a split screen (Fig. 13) is often used to show oppositions of ideas. The challenge is to automatically determine if the politicians are agreeing or in opposition. This would lead to the creation of negative ties. This often, but not always, occurs when the two politicians belong to opposing parties. Determining if the screen is split in half is challenging because they often appear with a similar background (Fig. 13). Currently, these links are typically investigated through selection of different parties in the captions.

\section{DISCUSSION AND FUTURE WORK}

We need to discuss the design study of our system at different levels, as proposed by Munzner [45]. From the domain and abstraction levels, we first need to provide more information for the different tasks necessary for users, and provide the correct pieces of information accordingly, at the idiom level to discuss our choices.

\section{A. Domain and abstraction}

Our goal is to provide quantitative and qualitative evidence of social phenomenon, such as the "presidentialization" of Japanese politics, from a video perspective. Our expert was amazed of the results of our system and its "explanatory power": the system is very good at capturing and explaining the changes in power, providing also a clue towards the representation of the opposition (e.g. the rise of Abe, the switch from LDP to DPJ, or the effect of the Great East earthquake). Although the main actors appear quite similar between cabinets - the main actors of the politico-media scene have not changed much between those cabinets - the organization of the scene itself is very different, displaying who plays a central role in NHK news.

There is still room for improvement to better investigate the question of opposition representation. Our system only provides partial answers regarding the roles of the main opposition members in the different networks. To fully determine if this is a bias of NHK, we need to extend our comparative analysis to other broadcasting channels and extend the survey until the present (the latest cabinets of Abe are also interesting to our domain expert, especially because of his strong ties with NHK). Fortunately, our analysis system can easily scale up to those challenges, the preprocessing time will only be a bit longer - although we may not be able to obtain the $10+$ years archive from different broadcasters.

After experiencing our system, our domain expert now wishes to tackle finer-grain observations by including stories when observing the timelines. Inspired by the wide-spread visual analytics discussed in Section II, this is an active direction for future work.

We worked on a limited number of given individuals that we were sure to be of interest; however, many other facetracks concern politicians or people "secondarily" involved in politics or young politicians on the rise. Our expert cannot give us a defined list of all political actors across all our archives. Towards this goal we would need to find a method for automatic annotation of public figures - something rather similar to 2016's Microsoft IRC Challenge MS-Celeb-1M [46].

Our system design is based on domain expert, but it incidentally helped us monitoring our video processing. The quality of face-tracking also has a strong impact on the trustfulness and strength of the observed results. One important goal of our end user is to reliably measure airtime, this will enable more direct comparisons and will provide straightforward answers to more of his domain questions related to the balance of power. However, he would need this measure to be accurate and stable across individuals. The rough approximation we can extract is not yet acceptable for this purpose. It varies too much from person to person, even on the same screen during the same news segment (Fig. 13). Moreover, we are very precise (we do not have incorrectly labelled face-tracks) but at a cost of a lower recall, and some face occurrences remain untracked. We can still only draw our conclusions because we are comparing large periods of time. We used deployable off-the-shelves techniques for face-tracking, but to enhance the trustfulness of the results, we need to update these techniques using the state-of-the art approaches, now based on deep learning ([28], [29], [27], [26]). The annotation phase is a bottleneck of our system as it is time consuming, and we are looking forward the improvement of large scale face recognition techniques [46].

The characterization of links is also an important aspect to consider. Broader detection, tracking, and recognition may certainly enable us to fully deploy a network of screenoverlapping interactions. However specific situations have a very different interpretation, and should be incorporated differently - such as the valence of ties when several actors are presented on a split screen or the same screen.

\section{B. Idiom}

Addressing Big Data's Variety is one task to which visual analytics has been very successful, most often using flexible 
visual re-encoding and linked-highlighting, for which the Tulip framework [43] is well adapted. It is however focused on tasks dedicated to graph manipulation and analysis. Important future work will certainly be a dedicated web based implementation.

Extending our data abstraction, as previously discussed, will have strong implications. Multiple visual encodings and interactions may be finely tuned and improved in the process (such as incorporating topics in the timeline visualization). We would like to dynamically coordinate social network exploration with timelines and video playback, also enriched with additional semantic data, and enable annotation.

The network exploration received the most positive feedback from our expert who found it useful to understand and explore his archive. It definitively seems promising to continue our investigation. Extension to other political actors should not go beyond a few hundred and our visual encoding should scale well. However, we are planning to explore a multiplex network representation when we include additional broadcast channels. We will then tackle interesting challenges in the coordination of the combined heterogeneous data.

\section{CONCLUSION}

We introduced the use of face-tracking data for visual analytics. With our system design, we discussed the entire pipeline to support our expert's analysis: how, from raw video data, we can create coordinated networks to explore the politico-media scene in an archive of 12 years of NHK's News 7, and access of individual video segments.

After introducing our domain expert's motivations and tasks, we presented the data abstraction we found appropriate from our previous work. We presented a top-level view of the different cabinets, network view of the activity of politicians in each cabinet, refined view of individual nodes and link occurrences in a timeline, and finally a coordinated video player that allows access to individual face-tracks inside individual videos. The combined views of the network and timelines provide interesting insights in the story behind the data, also isolating key actors in different periods (e.g. the rise of Abe, or the case of Ozawa). The view of centralities and weighted edges also brought up evidence of a presidentialization effect especially when the LDP is in power, even more obviously under Koizumi. Additionally, the general reader may take home the idea that, even without knowledge of the Japanese media landscape, one can quickly get an idea of who the main actors are and how they are interacting.

To our knowledge, no other system allows this type of exploration of news data, with comparison between parties in power through a focus on the interaction of politicians on screen. Of course, one can argue that this interaction could also be observed from text analysis using the same meta data. The extraction of visual information is an important parameter because images have a more powerful impact on viewers than semantic content. Other studies also harvested the explanatory power of network visualization as a main support for analysis but not for observing the interaction of politicians explaining the politico-media scene. Both views are not incompatible and in the end, our domain expert expressed the wish to characterize the relationships between politicians with semantic information for finer-grain analysis.

This work raises important challenges for visual analytics such as multiple coordination in different views when data are heterogeneous. Time representation remains another important challenge, for which we wish to go beyond timeline explorations. This will become especially true when we include multiple broadcasters and semantics for further comparison.

Currently only a design study, this work has given us useful directions that will help us improve our algorithms and design a more complete visual analytics system. The prototype is being currently used only by our expert in our lab; however, after improving it with more data and accurate analysis, we wish to deploy it to finally evaluate our investigation and conduct a comparative in-depth news analysis across multiple news broadcasters. Ultimately, this will enable the extraction of evidence to solve our expert's domain problems.

\section{ACKNOWLEDGMENTS}

The authors would like to thank Pr. Tetsuro Kobayashi from the City University of Hong Kong, the social scientist whose interest in the analysis of politics as presented by NHK motivated this research and whose feedbacks drove this system design. This work was conducted thanks to the Japanese Society for the Promotion of Science (as part of the FY2015 Grant-in-Aid for Scientific Research). We thank NHK for their allowing the use of their video data.

\section{REFERENCES}

[1] J. Allan, "Introduction to topic detection and tracking," pp. 1-16, 2002

[2] A. M. Barry, Visual intelligence: Perception, image, and manipulation in visual communication. SUNY Press, 1997.

[3] W. Jou and M. Endo, "Presidentialization of Japanese politics? examining political leader evaluations and vote choice," Japanese Journal of Political Science, vol. 16, no. 03, pp. 357-387, 2015.

[4] E. S. Krauss and B. Nyblade, "'presidentialization' in japan? the Prime Minister, media and elections in japan," British Journal of Political Science, vol. 35, no. 02, pp. 357-368, 2005.

[5] B. Renoust, T. Kobayashi, T. D. Ngo, D.-D. Le, and S. Satoh, "When face-tracking meets social networks: a story of politics in news videos," vol. 1, no. 1. Springer, 2016, pp. 1-25.

[6] Y. Lu, M. Steptoe, S. Burke, H. Wang, J.-Y. Tsai, H. Davulcu, D. Montgomery, S. R. Corman, and R. Maciejewski, "Exploring evolving media discourse through event cueing," Visualization and Computer Graphics, IEEE Transactions on, vol. 22, no. 1, pp. 220-229, 2016.

[7] N. Diakopoulos, M. Naaman, and F. Kivran-Swaine, "Diamonds in the rough: Social media visual analytics for journalistic inquiry," in IEEE Symposium on Visual Analytics Science and Technology (VAST), 2010. IEEE, 2010, pp. 115-122.

[8] M. Krstajic, M. Najm-Araghi, F. Mansmann, and D. A. Keim, "Incremental visual text analytics of news story development," in IS\&T/SPIE Electronic Imaging. International Society for Optics and Photonics, 2012, pp. 829407-829407.

[9] H. Li, B. Jou, J. G. Ellis, D. Morozoff, and S.-F. Chang, "News Rover: Exploring topical structures and serendipity in heterogeneous multimedia news," in Proceedings of the 21st ACM international conference on Multimedia. ACM, 2013, pp. 449-450.

[10] N. Hervé, M.-L. Viaud, J. Thièvre, A. Saulnier, J. Champ, P. Letessier, O. Buisson, and A. Joly, "Otmedia: the french transmedia news observatory," in Proceedings of the 21st ACM international conference on Multimedia. ACM, 2013, pp. 441-442.

[11] A. Kochtchi, T. v. Landesberger, and C. Biemann, "Networks of names: Visual exploration and semi-automatic tagging of social networks from newspaper articles," in Computer Graphics Forum, vol. 33, no. 3. Wiley Online Library, 2014, pp. 211-220.

[12] W. Kienreich and C. Seifert, "An application of edge bundling techniques to the visualization of media analysis results." pp. 375-380, 2010. 
[13] B. Renoust, G. Melancon, and T. Munzner, "Detangler: Visual analytics for multiplex networks," in Computer Graphics Forum, vol. 34, no. 3. Wiley Online Library, 2015, pp. 321-330.

[14] D. Benikova, U. Fahrer, A. Gabriel, M. Kaufmann, S. M. Yimam, T. von Landesberger, and C. Biemann, "Network of the day: Aggregating and visualizing entity networks from online sources," in Proceedings of the 12th KONVENS 2014, 2014.

[15] I. Ide and F. Nack, "Explain this to me!" ITE Transactions on Media Technology and Applications, vol. 1, no. 2, pp. 101-117, 2013.

[16] H. Luo, J. Fan, J. Yang, W. Ribarsky, and S. I. Satoh, "Exploring largescale video news via interactive visualization," in IEEE Symposium on Visual Analytics Science And Technology, 2006. IEEE, 2006, pp. 75-82.

[17] H. Luo, J. Fan, J. Yang, W. Ribarsky, and S. Satoh, "Analyzing largescale news video databases to support knowledge visualization and intuitive retrieval," in IEEE Symposium on Visual Analytics Science and Technology, 2007. IEEE, 2007, pp. 107-114.

[18] M. Itoh, M. Toyoda, C. Z. Zhu, S. Satoh, and M. Kitsuregawa, "Image flows visualization for inter-media comparison," in IEEE Pacific Visualization Symposium, 2014. IEEE, 2014, pp. 129-136.

[19] D. Lazer, "Networks in political science: Back to the future," $P S$ : Political Science \& Politics, vol. 44, no. 01, pp. 61-68, 2011.

[20] M. C. Waumans, T. Nicodème, and H. Bersini, "Topology analysis of social networks extracted from literature," PloS one, vol. 10, no. 6, p. e0126470, 2015.

[21] C.-J. Nan, K.-M. Kim, and B.-T. Zhang, "Social network analysis of TV drama characters via deep concept hierarchies," in Proceedings of ASONAM 2015, 2015

[22] N. Henry and J.-D. Fekete, "MatLink: Enhanced matrix visualization for analyzing social networks," in IFIP Conference on Human-Computer Interaction. Springer, 2007, pp. 288-302.

[23] N. Henry, J. D. Fekete, and M. J. McGuffin, "NodeTrix: A hybrid visualization of social networks," IEEE Transactions on Visualization and Computer Graphics, vol. 13, no. 6, pp. 1302-1309, Nov 2007.

[24] M. Ghoniem, J.-D. Fekete, and P. Castagliola, "A comparison of the readability of graphs using node-link and matrix-based representations," in Proceedings of the IEEE Symposium on Information Visualization (InfoVis). IEEE, 2004, pp. 17-24.

[25] M. Worring, A. Engl, and C. Smeria, "A multimedia analytics framework for browsing image collections in digital forensics," in Proceedings of the 20th ACM international conference on Multimedia. ACM, 2012, pp. 289-298.

[26] Y. Taigman, M. Yang, M. Ranzato, and L. Wolf, "Deepface: Closing the gap to human-level performance in face verification," in Proceedings of the IEEE Conference on Computer Vision and Pattern Recognition, 2014, pp. 1701-1708

[27] F. Schroff, D. Kalenichenko, and J. Philbin, "FaceNet: A unified embedding for face recognition and clustering," pp. 815-823, 2015.

[28] S. S. Farfade, M. J. Saberian, and L. Li, "Multi-view face detection using deep convolutional neural networks," CoRR, vol. abs/1502.02766, 2015.

[29] A. Ranftl, F. Alonso-Fernandez, and S. Karlsson, "Face tracking using optical flow," in Biometrics Special Interest Group (BIOSIG), 2015 International Conference of the. IEEE, 2015, pp. 1-5.

[30] T. D. Ngo, H. T. Vu, L. Duy-Dinh, and S. Satoh, "Face retrieval in large-scale news video datasets," IEICE Transactions on Information and Systems, vol. 96, no. 8, pp. 1811-1825, 2013.

[31] M. Sedlmair, M. Meyer, and T. Munzner, "Design study methodology: Reflections from the trenches and the stacks," IEEE Transactions on Visualization and Computer Graphics, vol. 18, no. 12, pp. 2431-2440, 2012.

[32] N. Katayama, H. Mo, I. Ide, and S. Satoh, "Mining large-scale broadcast video archives towards inter-video structuring," Advances in Multimedia Information Processing-PCM 2004, vol. 1, no. 1, pp. 489-496, 2005.

[33] I. Ide, H. Mo, N. Katayama, and S. Satoh, "Topic threading for structuring a large-scale news video archive," Image and Video Retrieval, vol. 1 , no. 1, pp. 123-131, 2004

[34] P. Viola and M. J. Jones, "Robust real-time face detection," International Journal of Computer Vision, vol. 57, no. 2, pp. 137-154, 2004.

[35] J. Shi and C. Tomasi, "Good features to track," in IEEE Computer Society Conference on Computer Vision and Pattern Recognition, 1994. IEEE, 1994, pp. 593-600.

[36] M. Everingham, J. Sivic, and A. Zisserman, "Hello! My name is... buffy"-automatic naming of characters in TV video." in $B M V C$, vol. 2, no. 4, 2006, p. 6.

[37] B. Shneiderman, "The eyes have it: A task by data type taxonomy for information visualizations," in IEEE Symposium on Visual Languages, 1996. IEEE, 1996, pp. 336-343.
[38] M. Brehmer and T. Munzner, "A multi-level typology of abstract visualization tasks," IEEE Transactions on Visualization and Computer Graphics, vol. 19, no. 12, pp. 2376-2385, 2013.

[39] M. Harrower and C. Brewer, "Colorbrewer.org: An online tool for selecting colour schemes for maps," The Cartographic Journal, vol. 40, no. 1 , pp. 27-37, 2003.

[40] A. Frick, A. Ludwig, and H. Mehldau, "A fast adaptive layout algorithm for undirected graphs (extended abstract and system demonstration)," in International Symposium on Graph Drawing. Springer, 1994, pp. 388403.

[41] J. Viard, M. Latapy, and C. Magnien, "Computing maximal cliques in link streams," Theoretical Computer Science, vol. 609, pp. 245-252, 2016.

[42] G. Shrey, D. Maxime, M. Michael, and K. Thomas, "MovementSlicer: Better Gantt charts for visualizing behaviors and meetings in movement data," in The 9th IEEE Pacific Visualization Symposium, PacificVis 2016. IEEE, 2016

[43] D. Auber, D. Archambault, R. Bourqui, M. Delest, J. Dubois, B. Pinaud, A. Lambert, P. Mary, M. Mathiaut, and G. Melançon, "Tulip III," Encyclopedia of Social Network Analysis and Mining, vol. 1, no. 1, pp. 2216-2240, 2014.

[44] M. Bostock, V. Ogievetsky, and J. Heer, "D3: Data-driven documents," IEEE Transactions on Visualization and Computer Graphics, vol. 17, no. 12 , pp. 2301-2309, 2011

[45] T. Munzner, "A nested model for visualization design and validation," IEEE Transactions on Visualization and Computer Graphics, vol. 15, no. 6, pp. 921-928, 2009 .

[46] Y. Guo, L. Zhang, Y. Hu, X. He, and J. Gao, "Ms-celeb-1m: A dataset and benchmark for large-scale face recognition," arXiv preprint arXiv:1607.08221, 2016

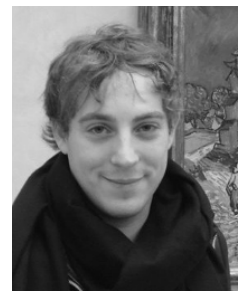

Benjamin Renoust is a JSPS post-doctoral researcher at the National Institute of Informatics (NII), Japan and at the CNRS UMI 3527 JapaneseFrench Laboratory for Informatics (JFLI), Japan. He was a research engineer at the National Audiovisual Institute (Ina) in Paris, France from 2009 to 2012, and received his $\mathrm{PhD}$ in 2014 from the University of Bordeaux, France. His research interests include visual analytics, network visualization, multimedia analytics, and network analysis. He is a member of IEEE-CS

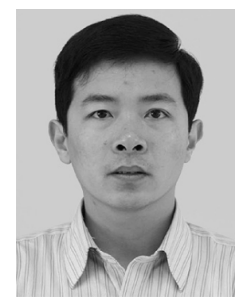

Duy-Dinh Le received his BS and MS degrees in 1995 and 2001, from the University of Science, Ho Chi Minh City, Vietnam, and his PhD degree in 2006 from The Graduate University for Advanced Studies (SOKENDAI), Japan. He is currently an associate professor at the National Institute of Informatics (NII), Japan. His research interests include semantic concept detection, video analysis and indexing, pattern recognition, machine learning and data mining.

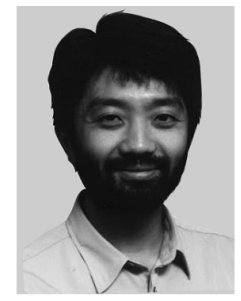

Shin'Ichi Satoh is a professor at the National Institute of Informatics (NII), Japan. He received his BE degree in 1987 and his ME and PhD degrees in 1989 and 1992 from the University of Tokyo. His research interests include video analysis and multimedia databases. He was a visiting scientist at the Robotics Institute, Carnegie Mellon University from 1995 to 1997 . He is a member of IPSJ, ITEJ, IEEE-CS, and ACM. 